\title{
PERFORMANCE INVESTIGATION ON THE HIGH-RESOLUTION WIDE-SWATH SAR SYSTEM OPERATING IN STRIPMAP QUAD-POL AND ULTRA-WIDE SCANSAR MODE
}

\author{
Federica Bordoni, Marwan Younis, Gerhard Krieger
}

German Aerospace Centre (DLR), Microwaves and Radar Institute, 82234 Wessling, Germany

\begin{abstract}
The High-Resolution Wide-Swath (HRWS) is the spaceborne Synthetic Aperture Radar system, promoted by the German Aerospace Centre, conceived to obtain simultaneously high spatial resolution and width swaths. Its main distinctive features are the multichannel architecture, the use of Digital Beamforming on receive and Scan on Receive algorithm. The HRWS allows for a spatial resolution of $1 \mathrm{~m}$ over swaths $70 \mathrm{~km}$ wide, in the basic Stripmap mode. In this paper, a performance investigation of the advance operational modes, Stripmap quadrature polarimetric and Ultra-Wide ScanSAR, is presented. The main design constraints are discussed, the proposed solutions explained and the achievable SAR performance numerically analyzed.
\end{abstract}

Index Terms- SAR, HRWS, DBF, SCORE

\section{INTRODUCTION}

The current generation of spaceborne Synthetic Aperture Radar (SAR) systems suffers a basic limitation in answering the final user needs: high spatial resolution and wide coverage cannot be attained simultaneously. For instance, a resolution around $1 \mathrm{~m}$ can be achieved over a swath width of $10 \mathrm{~km}$; whereas a coverage of $100 \mathrm{~km}$ is associated with a resolution in the order of $16 \mathrm{~m}$.

The importance to overcome this limitation has motivated an intensive research on new, more flexible SAR systems, whose distinctive characteristic is the use of multiple transmit/receive $(\mathrm{Tx} / \mathrm{Rx})$ channels and of processing techniques, such as the Digital Beamforming (DBF) [1-6]. This allows for a greater freedom in the system design, an improved imaging quality and, above all, a mitigation of the trade-off between swath width and spatial resolution [1-6].

Among these new SAR systems, the High-Resolution WideSwath (HRWS), promoted by the German Aerospace Centre (DLR), merges the advantages of an extensive illumination capability with the sensitivity provided by an antenna with high gain and directivity, and combines the flexibility of a multichannel architecture with a limited download data volume [6-8]. The HRWS SAR system is based on the SCan-On-REceive (SCORE) algorithm: a large swath is illuminated by using a wide, static transmit beam; whereas in reception the DBF is employed in order to obtain a sharp and high gain pattern, which scans the swath according to the expected direction of arrival of the backscattered SAR pulse [6]. The conflict between swath width and azimuth resolution is overcome by using multiple phase centers located along the azimuth direction, according to the Displaced Phase Center Antenna (DPCA) technique, whose feasibility is extended through the application of the multiaperture reconstruction algorithm $[1,4]$.

The SAR performance of the HRWS system operating in Stripmap, single polarimetric (single-pol) mode was presented [7] and further analyzed in [8], showing the possibility to achieve a spatial resolution of $1 \mathrm{~m}$ over swaths $70 \mathrm{~km}$ wide. In this paper, for the first time, a performance investigation in Stripmap, quadrature polarimetric (quadpol) mode and in Ultra-Wide (UW) ScanSAR mode is presented. In particular, for each operational mode, the main design constraints are clarified, the proposed solutions explained and the achievable SAR performance numerically analyzed.

\section{REFERENCE HRWS SAR SYSTEM}

The reference HRWS SAR system, described in Table 1, has been considered for the performance investigation.

\begin{tabular}{|l|l|}
\hline Parameter & Value \\
\hline Orbit height & $520 \mathrm{~km}$ \\
\hline Access range (inc. angle) & $20^{\circ}-50^{\circ}$ \\
\hline RF centre frequency & $9.600 \mathrm{GHz}$ \\
\hline Average Tx power & $2171 \mathrm{~W}$ \\
\hline Trans. bandwidth & $<=408 \mathrm{MHz}$ \\
\hline Total losses & $8.38 \mathrm{~dB}$ \\
\hline Antenna height & $1.06 \mathrm{~m}$ \\
\hline Antenna length & $8.75 \mathrm{~m}$ \\
\hline Nr. elev. Tx x Rx channels & $1 \times 12$ \\
\hline Nr. az. Tx x Rx channels & $1 \times 7$ \\
\hline Polarization & Full pol. \\
\hline
\end{tabular}

Tab. 1. Parameters of the reference HRWS SAR system.

The HRWS is a monostatic system, based on a planar phased array antenna architecture. The antenna has a size of $1.06 \mathrm{~m} \times 8.75 \mathrm{~m}$ (high $\times$ length) and is uniformly divided along both directions in singular radiating elements. The 
DPCA is implemented by using 7 phase centers, uniformly displaced along the antenna length. The large elevation size of the antenna is associated with a sharp receive elevation pattern; SCORE algorithm is implemented by using 12 channels, uniformly displaced along the antenna high. In transmission, a broadening of the elevation and azimuth patterns is obtained by the Phase Spoiling technique [8].

\section{STRIPMAP QUAD-POL MODE}

Strict design constraints and performance requirements characterize a multichannel SAR system operating in quadpol mode. In fact, the pulse repetition frequency (PRF) should be approximately doubled with respect to the PRF used in single-pol mode, in order to preserve the azimuth resolution. The use of a higher PRF increases the number and reduces the extension of the subswaths necessary to cover the full access range, according to the timing diagram. Moreover, it augments the number of range ambiguities, which become close, especially in far range. This makes difficult to meet the RASR (range ambiguity-to-signal ratio) requirements, above all on the cross-pol image, and imposes a severe limitation of the AASR (azimuth ambiguity-tosignal ratio) in order to keep low the overall ambiguity level. These constraints are even more rigid for a multichannel system with respect to a conventional system, due to its lower degrees of freedom in the choice of the PRF. In fact, the multichannel system should operate with a PRF close to the uniform PRF, in order to limit possible performance degradation associated with the multichannel processing [4]. Accordingly, the option to employ a somewhat lower PRF at the cost of a degraded resolution is not attractive.

Figure 1 shows the timing diagram and the subswaths considered for performance analysis. The overall access range is covered by 11 subswaths, whose width varies between 20 and $50 \mathrm{~km}$ (in single-pol mode the corresponding number of subswaths is 6 and their width varies between 70 and $80 \mathrm{~km}$ ). The PRF values of each subswaths have a deviation from the uniform PRF value $(3460 \mathrm{~Hz})$ not greater than $2 \%$, except for the $2^{\text {nd }}$ subswath, whose deviation is $9 \%$.

The use of multiple channels provides flexibility in the design of the elevation patterns. In fact, it allows to adapt the mainlobe width of the transmit elevation pattern to the subswath size and to control the side lobe level (SLL). In particular, the solution here proposed is to employ the Phase Spoiling technique in transmission, in order to broaden the mainlobe of the patterns illuminating the near range subswaths $\left(1^{\text {st }}-5^{\text {th }}\right)$, and a Hamming widowing in reception, in order to reduce the SLL. Figure 2 shows the elevation patterns in the $2^{\text {nd }}$ subswath; the direction of arrival of the useful signal and of the range ambiguities is also evidenced.

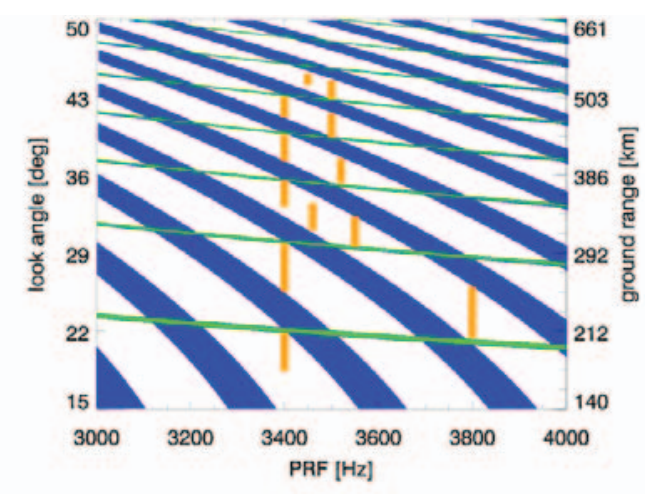

Fig. 1. Stripmap quad-pol: timing diagram. The selected subswaths are indicated by the vertical segments.

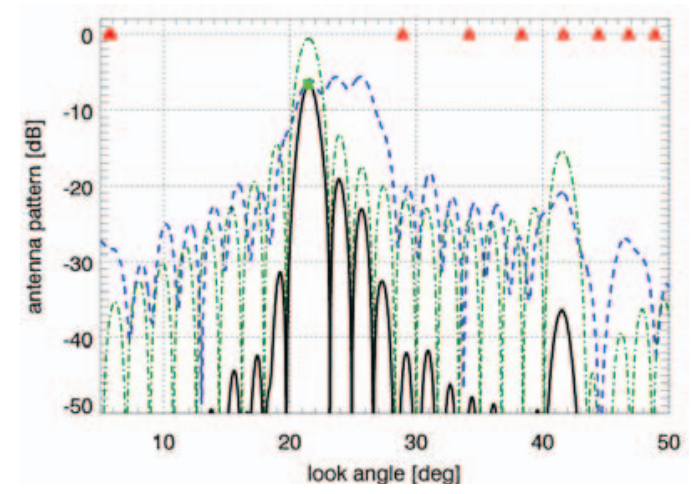

Fig. 2. Elevation patterns in the $2^{\text {nd }}$ subswath (solid line: 2 -way, dashed: transmit, dash-dot: receive). The direction of arrival of the signal an of the ambiguities are indicated by the symbols.

The numerical analysis is developed by considering a requirement on the spatial resolution (azimuth and range) of $1 \mathrm{~m}$. Figure 3 shows the RASR in the cross-pol case: it is below $-19 \mathrm{~dB}$ over all the access range. In co-pol, the RASR is below $-27 \mathrm{~dB}$.

The AASR, shown in Figure 4, is always below $-30.5 \mathrm{~dB}$, and consequently negligible with respect to the RASR in cross-pol.

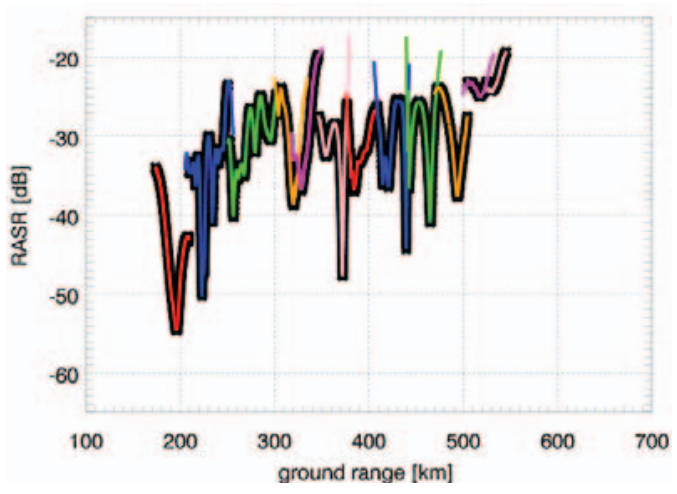

Fig. 3. Stripmap quad-pol, cross-pol: RASR vs. ground range. 


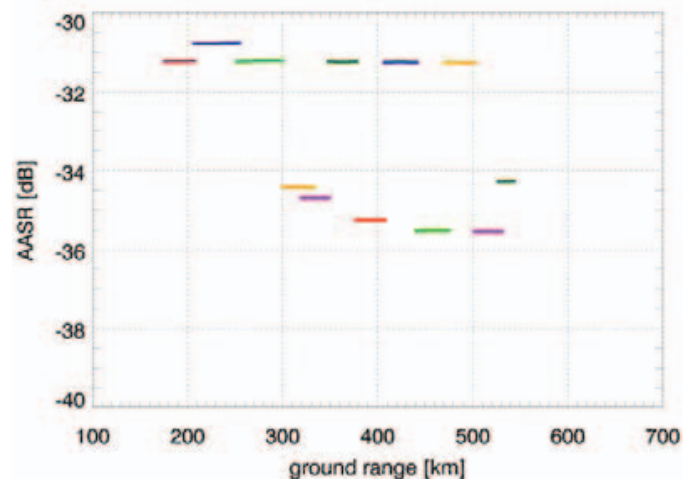

Fig. 4. Stripmap quad-pol: AASR vs. ground range.

In Figure 5, the NESZ is reported: it is below $-19.5 \mathrm{~dB}$, with a variation within the subswath determined mainly by the transmit pattern shape. Finally, Figure 6 shows the normalized SNR Scaling [4]: over the complete Doppler bandwidth, it is close to the optimum value of $0 \mathrm{~dB}$ in all the subswaths, except the $2^{\text {nd }}$, where the PRF deviation from the uniform value is higher; over the processed bandwidth, all the subswaths show approximately the same improvement.

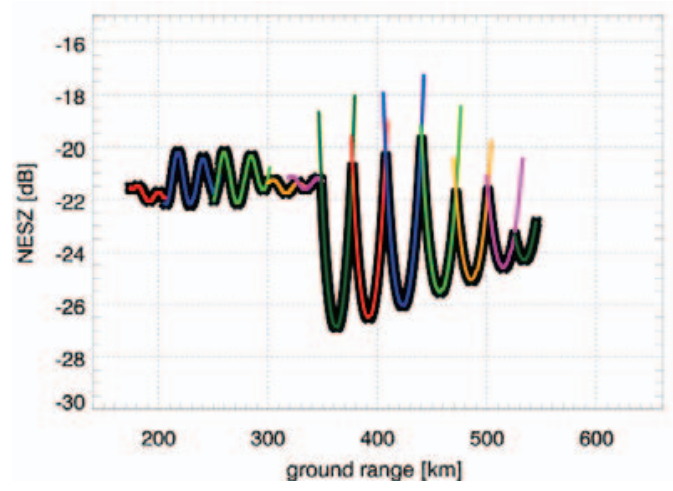

Fig. 5. Stripmap quad-pol: NESZ vs. ground range.

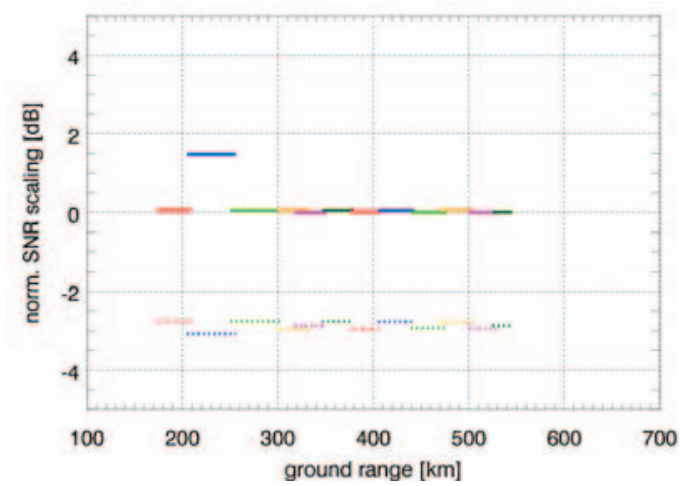

Fig. 6. Stripmap quad-pol: normalized SNR Scaling vs. ground range, computed over the total unambiguous Doppler bandwidth (solid line) and over the processed Doppler bandwidth (dotted).

\section{ULTRA-WIDE SCANSAR MODE}

The UW ScanSAR mode is conceived in order to maximize the swath width: the full access range of $375 \mathrm{~km}$ is imaged in a single pass. This allows for a complete imaging of the Earth with a repeat time of 8 days.

Figure 7 shows the timing diagram, considered for performance analysis: the access range is covered by 6 overlapping subswaths, each one associated to a specific burst and PRF value.

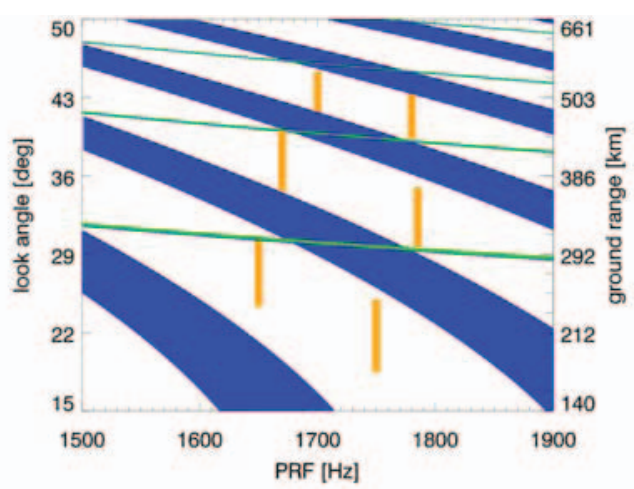

Fig. 7. UW ScanSAR: timing diagram. The selected subswaths are indicated by the vertical segments.

In the UW ScanSAR mode, the azimuth pattern design has a key influence on the SAR performance. In particular, the 2way pattern should be simultaneously characterized by: (i) wide mainlobe, to achieve the best azimuth resolution; (ii) low ripple level within the mainlobe, to reduce the scalloping; (iii) high mainlobe level, to meet the NESZ requirements; (iv) low sidelobe level, to control the AASR. The design solution here proposed utilizes the Phase Spoiling technique to synthesize the azimuth transmit patter. Figure 8 shows the utilized azimuth patters versus the Doppler frequency.

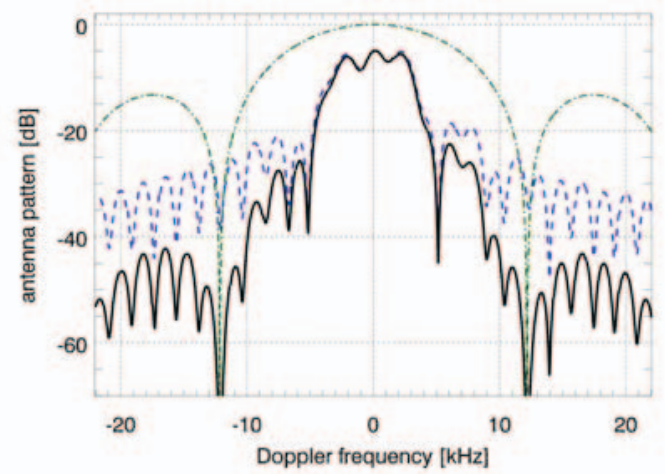

Fig. 8. UW ScanSAR: azimuth patterns (solid line: 2-way, dashed: transmit, dash-dot: receive). 
The numerical analysis, developed by considering a range resolution equal to $1 \mathrm{~m}$, demonstrates the possibility to obtain the following SAR performance in UW ScanSAR mode: azimuth resolution below $8.7 \mathrm{~m}$; AASR below -24 $\mathrm{dB}$; scalloping below $2.1 \mathrm{~dB}$; RASR below $-28 \mathrm{~dB}$; NESZ below $-22.6 \mathrm{~dB}$.

Figure 9 and 10 show the AASR and scalloping in the different subswaths versus the Doppler centre, respectively. In particular, the comparison between Figure 10 and Figure 8 evidences the influence on the scalloping performance of the ripples within the pattern mainlobe.

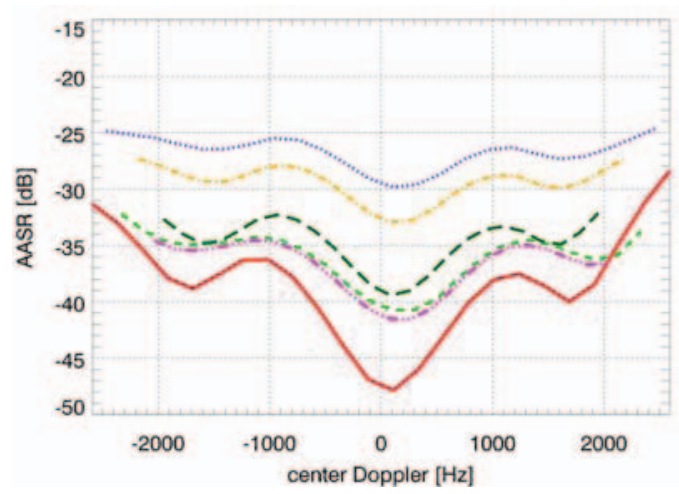

Fig. 9. UW ScanSAR: AASR vs. Doppler center in each subswath.

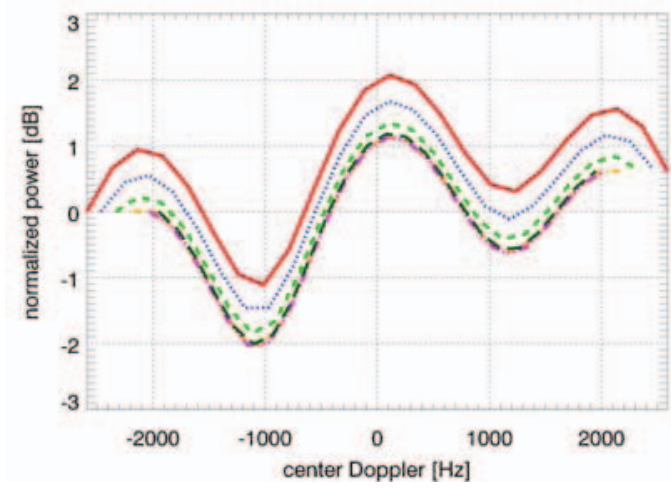

Fig. 10. UW ScanSAR: scalloping vs. Doppler center in each subswath.

\section{CONCLUSIONS}

A performance investigation on the HRWS SAR system operating in Stripmap quad-pol and Ultra-Wide (UW) ScanSAR mode has been presented.

In Stripmap quad-pol mode, the choice of the PRF is associated with challenging design constraints and performance requirements. The proposed solution takes advantage of the flexibility offered by the multichannel architecture of the system and is based on the use of the Phase Spoiling technique and Hamming windowing for elevation pattern generation. The numerical analysis shows the possibility to achieve the following SAR performance: azimuth and range resolution equal to $1 \mathrm{~m}$; swath width between 20 and $50 \mathrm{~km}$; RASR below $-19.5 \mathrm{~dB}$ in cross-pol and below $-27 \mathrm{~dB}$ in co-pol; AASR below $-30.5 \mathrm{~dB}$; NESZ below $-19 \mathrm{~dB}$.

In UW ScanSAR mode, the driving requirement is on the swath width, which is about $375 \mathrm{~km}$. This allows imaging the entire Earth with a repeat time of 8 days. To meet this objective, the design of the azimuth pattern plays a key role. The proposed solution uses the Phase Spoiling to synthesize the transmit pattern. The reported analysis, performed by assuming a range resolution equal to $1 \mathrm{~m}$, demonstrates the possibility to obtain a swath width of $375 \mathrm{~km}$ with an azimuth resolution below $9 \mathrm{~m}$; AASR under $-24 \mathrm{~dB}$; scalloping under $-2.1 \mathrm{~dB}$; RASR under $-28 \mathrm{~dB}$; NESZ under $-22.6 \mathrm{~dB}$.

\section{REFERENCES}

[1] G. Krieger, N. Gebert, A. Moreira, "Unambiguous SAR signal reconstruction from nonuniform displaced phase center sampling", IEEE Trans. Geosc. and Remote Sens. Letters, Vol. 1 , No. 4, pp. $260-264$, Oct. 2004.

[2] G. Krieger, N. Gebert, A. Moreira, "Multidimensional Waveform Encoding: A New Digital Beamforming Technique for Synthetic Aperture Radar Remote Sensing", IEEE Trans. on Geosci. and Remote Sens., vol. 46, no. 1, pp.31-46, Jan. 2008.

[3] M. Younis, F. Bordoni, N. Gebert, G. Krieger, "Smart MultiAperture Radar Techniques for Spaceborne Remote Sensing", Proc. IEEE Int. Geosci. and Remote Sens. Symp., IGARSS, vol. 3, pp. 278-280, Boston, MA, USA, July 2008.

[4] N. Gebert, G. Krieger, and A. Moreira, "Digital Beamforming on Receive: Techniques and Optimization Strategies for HighResolution Wide-Swath SAR Imaging", IEEE Trans. on Aerosp. and Electron. Syst., Vol. 45, No. 2, pp. 564-592, April 2009.

[5] G. Krieger, M. Younis, N. Gebert, S. Huber, F. Bordoni, A. Patyuchenko, A. Moreira, "Advanced Digital Beamforming Concepts for Future SAR Systems", Proc. IEEE Int. Geosci. and Remote Sens. Symp., IGARSS, Honolulu, Hawaii, USA, July 2010.

[6] M. Suess, B. Grafmueller, R. Zahn, "A Novel High Resolution, Wide Swath SAR System”, Proc. IEEE Geosci. and Remote Sens. Symp., IGARSS, vol. 3, pp. 1013-1015, Sydney, NSW, Australia, July 2001.

[7] C. Fischer, C. Heer, G. Krieger, R. Werninghaus, "A High Resolution Wide Swath SAR", Proc. European Conf. on Synthetic Aperture Radar, EUSAR, Dresden, DE, May 2006.

[8] F. Bordoni, M. Younis, N. Gebert, G. Krieger, C. Fischer, "Performance Investigation on the High-Resolution Wide-Swath SAR System with Monostatic Architecture", Proc. of the European Conf. on Synthetic Aperture Radar, EUSAR, pp. 11221125, Aachen, Germany, June 2010. 